\title{
INDEPENDENT COMPONENT ANALYSIS
}

THEORY AND APPLICATIONS 


\section{INDEPENDENT COMPONENT ANALYSIS}

THEORY AND APPLICATIONS

by

\section{TE-WON LEE}

Computational Neurobiology Laboratory

The Salk Institute, La Jolla, California 
A C.I.P. Catalogue record for this book is available from the Library of Congress.

ISBN 978-1-4419-5056-7 ISBN 978-1-4757-2851-4 (eBook) DOI 10.1007/978-1-4757-2851-4

Printed on acid-free paper

All Rights Reserved

(C) 1998 Springer Science+Business Media Dordrecht

Originally published by Kluwer Academic Publishers in 1998

Softcover reprint of the hardcover 1st edition 1998

No part of the material protected by this copyright notice may be reproduced or

utilized in any form or by any means, electronic or mechanical,

including photocopying, recording or by any information storage and

retrieval system, without written permission from the copyright owner. 
This book is dedicated to my parents Jeong-Bog Lee and Sun-Ja Kang, my sister Soon-Hie and my brother Yu-Won. 


\section{Contents}

Abstract $\quad$ xi

Preface $\quad$ xiii

Acknowledgments $\quad$ xvii

List of Figures $\quad$ xix

List of Tables $\quad$ xxiii

Abbreviations and Symbols $\quad$ XXV

Introduction $\quad$ xxix

Part I Independent Component Analysis: Theory

1. BASICS 5

1.1 Overview 5

1.2 Bayesian Probability Theory 6

1.3 Information Theory $\quad 7$

$\begin{array}{ll}\text { 1.3.1 Differential Entropy } & 10\end{array}$

1.3.2 Maximum Entropy 11

1.4 Artificial Neural Networks 13

$\begin{array}{lll}\text { 1.4.1 Neural networks using unsupervised learning rules } & 14\end{array}$

1.4.2 The Principle of Maximum Entropy Preservation 18

1.5 Higher-Order Statistics $\quad 21$

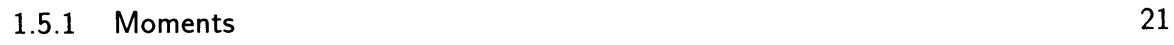

$\begin{array}{lll}1.5 .2 & \text { Cumulants } & 23\end{array}$

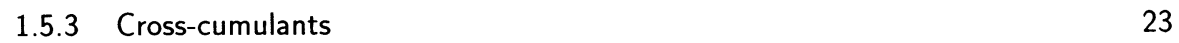

$\begin{array}{lll}1.6 & \text { Summary } & 24\end{array}$

2. INDEPENDENT COMPONENT ANALYSIS $\quad 27$

$\begin{array}{lll}2.1 & \text { Overview } & 27\end{array}$

2.2 Problem statement and assumptions 29

2.3 The Poverty of PCA 31 
2.4 The Information Maximization Approach to ICA 35

2.5 Derivation of the Infomax Learning Rule for ICA 37

$\begin{array}{lll}2.6 & \text { A simple but general ICA learning rule } & 42\end{array}$

2.6.1 Deriving the extended infomax learning rule to separate sub- and superGaussian sources 43

$\begin{array}{ll}\text { 2.6.2 Switching between nonlinearities } & 47\end{array}$

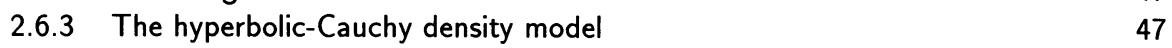

$\begin{array}{llr}2.7 & \text { Simulations } & 49\end{array}$

$\begin{array}{lll}2.7 .1 & 10 \text { Mixed Sound Sources } & 49\end{array}$

2.7.2 20 Mixed Sound Sources 52

2.8 Convergence properties in blind source separation 56

2.8.1 An intuition for the natural gradient 56

2.8.2 Robustness to parameter mismatch 58

$\begin{array}{lll}2.9 & \text { Discussions } & 62\end{array}$

2.9.1 Comparison to other algorithms and architectures 62

$\begin{array}{ll}\text { 2.9.2 Applications to real world problems } & 62\end{array}$

$\begin{array}{ll}2.9 .3 & \text { Biological plausibility } \\ 2.9 .4 & 63\end{array}$

$\begin{array}{ll}2.9 .4 & \text { Limitations and future research }\end{array}$

$\begin{array}{lll}2.9 .5 & \text { Conclusions } & 64\end{array}$

3. A UNIFYING INFORMATION-THEORETIC FRAMEWORK FOR ICA 67

$\begin{array}{lll}3.1 & \text { Overview } & 67\end{array}$

$\begin{array}{lll}3.2 & \text { Information Maximization } & 68\end{array}$

$\begin{array}{ll}3.3 \text { Negentropy Maximization } & 69\end{array}$

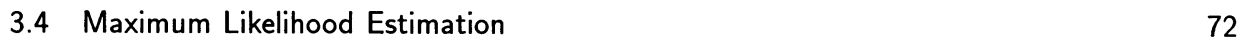

$\begin{array}{lll}3.5 & \text { Higher-order moments and cumulants } & 73\end{array}$

$\begin{array}{lll}3.6 & \text { Nonlinear PCA } & 76\end{array}$

$\begin{array}{lll}3.7 & \text { Bussgang Algorithms } & 78\end{array}$

$\begin{array}{ll}3.8 \text { Conclusion } & 80\end{array}$

4. BLIND SEPARATION OF TIME-DELAYED AND CONVOLVED SOURCES 83

$\begin{array}{lll}4.1 & \text { Overview } & 83\end{array}$

4.2 Problem statement and assumptions $\quad 85$

4.3 Feedback Architecture $\quad 86$

$\begin{array}{lll}\text { 4.3.1 Learning Rules } & 87\end{array}$

$\begin{array}{lll}\text { 4.3.2 Simulations } & 89\end{array}$

4.4 Feedforward Architecture 90

4.4.1 Learning Rules $\quad 92$

$\begin{array}{ll}\text { 4.4.2 Simulations } & 94\end{array}$

4.5 Experiments in real environments $\quad 94$

4.5.1 Speech Recognition Results 101

$\begin{array}{lll}4.6 & \text { Bussgang algorithms } & 101\end{array}$

$\begin{array}{ll}\text { 4.7 Time-delayed decorrelation methods } & 102\end{array}$

$\begin{array}{lll}\text { 4.7.1 Experimental Results with TDD } & 103\end{array}$

$\begin{array}{ll}\text { 4.7.2 Discussions on TDD } & 105\end{array}$

$\begin{array}{lll}4.8 & \text { Spectrogram ICA } & 105\end{array}$ 
$\begin{array}{lll}4.9 & \text { Conclusions } & 106\end{array}$

$\begin{array}{ll}\text { 4.9.1 Future Research } & 107\end{array}$

5. ICA USING OVERCOMPLETE REPRESENTATIONS 111

5.1 Learning Overcomplete Representations 113

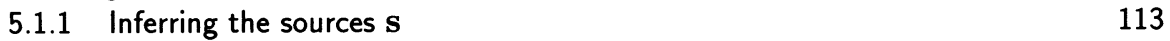

5.1.2 Learning the basis vectors $\mathbf{A} \quad 113$

5.2 Experimental Results 114

$\begin{array}{lll}\text { 5.2.1 Blind Separation of Speech Signals } & 114\end{array}$

5.2.2 Blind Separation of Speech and Music Signals 116

$\begin{array}{ll}\text { 5.2.3 Preliminary results with other mixtures } & 117\end{array}$

$\begin{array}{lll}5.3 & \text { Discussion } & 117\end{array}$

$\begin{array}{lll}\text { 5.3.1 Comparison to other methods } & 117\end{array}$

$\begin{array}{ll}\text { 5.3.2 Conclusions } & 119\end{array}$

6. FIRST STEPS TOWARDS NONLINEAR ICA 123

$\begin{array}{ll}6.1 \text { Overview } & 123\end{array}$

6.2 A simple nonlinear mixing model $\quad 124$

$\begin{array}{ll}6.3 \text { Learning Algorithm } & 125\end{array}$

6.3.1 Learning Rules for Sigmoidal Nonlinear Mixing 126

6.3.2 Learning Rules for Flexible Nonlinearities 127

$\begin{array}{lll}6.4 & \text { Simulation Results } & 128\end{array}$

6.5 A linearization approach to nonlinear ICA 130

$\begin{array}{lll}6.6 & \text { Discussions } & 135\end{array}$

6.6.1 Other approaches to nonlinear ICA 135

$\begin{array}{ll}\text { 6.6.2 Conclusions and future research } & 137\end{array}$

Part II Independent Component Analysis: Applications

7. BIOMEDICAL APPLICATIONS OF ICA 145

$\begin{array}{lll}7.1 & \text { Overview } & 145\end{array}$

7.2 ICA of Electroencephalographic Data 147

7.2.1 Simple examples of applying ICA to EEG data 148

$\begin{array}{ll}\text { 7.3 EEG artifact removal using extended infomax } & 149\end{array}$

$\begin{array}{lll}\text { 7.3.1 Methods and Materials } & 155\end{array}$

$\begin{array}{ll}\text { 7.3.2 Discussion of EEG applications } & 157\end{array}$

7.4 Functional Magnetic Resonance Imaging Analysis $\quad 157$

$\begin{array}{lll}\text { 7.4.1 fMRI Methods } & 158\end{array}$

$\begin{array}{lll}\text { 7.4.2 fMRI Results } & 159\end{array}$

$\begin{array}{ll}\text { 7.4.3 Discussions and Conclusions on fMRI } & 160\end{array}$

$\begin{array}{ll}7.5 \text { Conclusions and future research } & 163\end{array}$

8. ICA FOR FEATURE EXTRACTION 167

$\begin{array}{lll}8.1 \text { Overview } & 167\end{array}$

8.2 ICA of natural images 168

8.3 ICA of natural images using extended infomax 169 
8.4 ICA for Feature Extraction $\quad 170$

$\begin{array}{ll}\text { 8.5 Discussions and Conclusions } & 173\end{array}$

9. UNSUPERVISED CLASSIFICATION WITH ICA MIXTURE MODELS 177

$\begin{array}{lll}9.1 & \text { Overview } & 177\end{array}$

9.2 The ICA Mixture Model 178

$\begin{array}{lll}9.3 & \text { Simulations } & 181\end{array}$

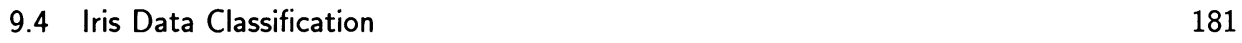

$\begin{array}{ll}9.5 \text { Conclusions } & 182\end{array}$

10. CONCLUSIONS AND FUTURE RESEARCH 187

$\begin{array}{ll}10.1 \text { Conclusions } & 187\end{array}$

$\begin{array}{ll}10.2 \text { Future Research } & 188\end{array}$

$\begin{array}{ll}\text { Bibliography } & 193\end{array}$

$\begin{array}{ll}\text { About the author } & 207\end{array}$

Index 
Independent Component Analysis (ICA) is a signal processing method to extract independent sources given only observed data that are mixtures of the unknown sources. Recently, blind source separation by ICA has received attention because of its potential signal processing applications such as speech enhancement systems, telecommunications and medical signal processing.

This book presents theories and applications of ICA. Based on theories in probabilistic models, information theory and artificial neural networks several unsupervised learning algorithms are presented that can perform ICA. The seemingly different theories such as infomax, maximum likelihood estimation, negentropy maximization, nonlinear PCA, Bussgang algorithm and cumulant-based methods are reviewed and put in an information theoretic framework to unify several lines of ICA research (Lee et al., 1998a). An extension of the infomax algorithm of Bell and Sejnowski (1995) is presented that is able to blindly separate mixed signals with sub- and super-Gaussian source distributions (Girolami, 1997b; Lee et al., 1998b). The learning algorithms are furthermore extended to deal with the multichannel blind deconvolution problem. The use of filters allows the separation of voices recorded in a real environment (cocktail party problem). Several constraints in the ICA formulation such as the linear model assumption, the number of sensors and the low-noise assumption, are tackled with new methods. In particular, an overcomplete representation of the ICA formulation (Lewicki and Sejnowski, 1998c) whcih includes an additive noise model can be used to infer more sources than sensors.

The second part of the book presents signal processing applications of ICA to realworld problems. The ICA algorithm has been successfully applied to many biomedical signal processing problems such as the analysis of electroencephalographic (EEG) data (Makeig et al., 1997; Jung et al., 1998a) and functional magnetic resonance imaging (fMRI) data (McKeown et al., 1998b). Bell and Sejnowski (1997) suggested that independent components of natural scenes are edge filters. Those independent image components can be used as features in pattern classification problems such as visual lip-reading and face recognition systems. The ICA algorithm can be furthermore embedded in an expectation maximization framework with the goal to classify clusters of ICA models. This approach is an extension of the Gaussian mixture model for non-Gaussian priors. Results on classification benchmarks demonstrate that ICA cluster models can improve classification results.

ICA is a fairly new and a generally applicable method to several theoretical and practical challenges in signal processing. Successful results in EEG, fMRI, speech enhancement and face recognition systems demonstrate its power and its wide range of potential applications. 


\section{Preface}

At a meeting held on April 13-16, 1986 in Snowbird Utah on Neural Networks for Computing Jeanny Herault and Christian Jutten (Herault and Jutten, 1986) contributed a research paper entitled "Space or time adaptive signal processing by neural network models". They presented a recurrent neural network model and a learning algorithm based on a version of the Hebb learning rule that, they claimed, was able to blindly separate mixtures of independent signals. They demonstrated the separation of two mixed signals and also mentioned the possibility of unmixing stereoscopic visual signals with four mixtures. This paper opens a remarkable chapter in the history of signal processing, a chapter that is hardly more than 10 years old.

The problem of source separation is an old one in electrical engineering and has been well studied; many algorithms exist depending on the nature of the mixed signals. The problem of blind source separation is more difficult since without knowledge of the signals that have been mixed, it is not possible to design appropriate preprocessing to optimally separate them. The only assumption made by Herault and Jutten was independence, but additional constraints are needed on the probability distribution of the sources. If one assumes, as is often done, that the source signals are Gaussian, then it is easy to show that this problem has no general solution. Subsequent research has shown that the best performance was obtained by the Herault-Jutten network when the source signals were sub-Gaussian (Cohen and Andreou, 1992); that is, for signals whose kurtosis was less than that of a Gaussian distribution.

In the neural network field, this network model was overshadowed at the time by the more popular Hopfield network, which would soon be eclipsed in popularity by the backpropagation algorithm for multilayer perceptrons. Nonetheless, a line of research was begun that only gradually made clear the true nature of the problem. As is often the case, what is important is not the specifics of the algorithm, but the way the problem is formulated. The general framework for independent component analysis introduced by Herault and Jutten is most clearly stated in Comon (1994). Within the signal processing community, a cornucopia of ever more sophisticated algorithms was developed based on cumulants, generalizing the third-order nonlinearity first used by Herault and Jutten. 
By 1994 the forefront of the neural network field had moved from supervised learning algorithms to unsupervised learning. A fast and efficient ICA algorithm was needed that could scale up with the size of the problem at least as well as backpropagation, which by this time was being used on networks with over a million weights. Anthony Bell in my laboratory was working on an infomax (Linsker, 1992) approach to ICA. Tony's first results were obtained using Mathematica and a version of his algorithm that depended on inverting a matrix (Bell and Sejnowski, 1995). This was probably fortunate since the long pauses during convergence gave him ample time to think about the problem and to benefit from vigorous discussions with Nicol Schraudolph and Paul Viola, who at the time were sharing an office with a wonderful view of the Pacific Ocean. Both Nici and Paul were working on problems that involved estimating entropy gradients, so there was a keen competition to see whose algorithm would perform best. During this period, Tony collaborated by long-distance with Te-Won Lee, who at the time was visiting Carnegie- Mellon University, on blind source separation of acoustically recorded sound mixtures, taking into account time delays.

Amari (1997a) soon realized that the infomax ICA algorithm could be improved by using the natural gradient, which multiplies the gradient of the feedforward weight matrix $\mathbf{W}$ by a positive definite matrix $\mathbf{W}^{T} \mathbf{W}$, and speeds up the convergence by eliminating the matrix inversion. This improvement, which was independently discovered by Cardoso and Laheld (1996), allows infomax ICA to be scaled up and makes it a practical algorithm for a variety of real-world problems. However, the original infomax ICA algorithm was only suitable for super-Gaussian sources. Te-Won Lee realized that a key to generalizing the infomax algorithm to arbitrary non-Gaussian sources was to estimate moments of the source signals and to switch the algorithm appropriately. In collaboration with Mark Girolami, who had been working on similar algorithms in the context of projection pursuit, he soon developed an efficient extended version of the infomax ICA algorithm (Lee, Girolami and Sejnowski, 1998) that is suitable for general non-Gaussian signals. All of these developments are presented in this book with a clarity that makes them accessible to anyone with an interest in ICA.

Another important component of this book is the comparison between several different approaches that have been taken to blind source separation, which include maximum likelihood, Bussgang methods based on cumulants, and projections pursuit and negentropy methods. Te-Won Lee shows that these are all closely related to the infomax framework (Lee, Girolami, Bell and Sejnowski, 1998). Thus, a large number of researchers who have attacked ICA from a variety of different directions are converging on a common set of principles and, ultimately, a well understood class of algorithms. There is still much work that is left to do. It is still true as Herault and Jutten mention in their 1986 paper, "We cannot prove convergence of this algorithm because of nonlinearity of the adaptation law and nonstationarity of the signals." We still do not have an adequate explanation for why ICA does converge for so many problems, almost always to the same solutions, even when the signals were not derived from independent sources. 
What makes this book especially valuable to the practitioner is that it also includes examples of several real-world applications. Although the blind separation of mixtures of prerecorded signals is a useful benchmark, a more challenging problem is to apply ICA to recordings of real-world signals for which the underlying sources, if any, are unknown. An important example is the application of extended infomax ICA to electroencephalographic (EEG) recordings of scalp potentials in humans. The electrical signals originating from the brain are quite weak at the scalp, in the microvolt range, and there are larger artifactual components arising from eye movements and muscles. It has been a difficult challenge to eliminate these artifacts without altering the brain signals. ICA is ideally suited to this task, since the brain and the scalp are good volume conductors and to a good approximation, the recordings are different linear mixtures of the brain signals and the artifacts. The extended infomax ICA algorithm has proven to be the best method yet for separating out these artifacts, which include sub-Gaussian sources such as $60 \mathrm{~Hz}$ line noise and blinks, from the brain signals, which are generally super-Gaussian (Jung et al., 1998a). The future of this algorithm looks quite bright for biomedical applications, including the analysis of extremely large datsets from functional Magnetic Resonance Imaging (fMRI) experiments (McKeown et al., 1998b).

ICA can be applied to many problems where mixtures are not orthogonal and the source signals are not Gaussian. Most information bearing signals have these characteristics. There are many interesting theoretical problems in ICA that have yet to be solved and there are many new applications, such as data mining, that have yet to be explored. The appearance of this book marks an important milestone in the maturation of ICA research. The theoretical framework developed here should provide a strong foundation for future research and applications. 


\section{Acknowledgments}

This book was made possible with the enormous support of many people. Most particularly Tony Bell and Terry Sejnowski gave guidance and advice from the beginning of my studies in ICA until now. The scientific collaboration and discussions I had with them are invaluable. Tony inspired me to think about ideas so thoroughly that he changed an engineering reasoning into a scientific mind. Working with Terry was like walking with an encyclopedia. His broad knowledge was fascinating and although he was constantly collaborating with many other researchers at the same time he was always accessible until late after midnight.

I was privileged to stay at the Salk Institute, the computational neurobiology lab. (CNL), and to work with the leading researchers in biomedical signal processing: Tzyy-Ping Jung, Martin McKeown and Scott Makeig. The combined force of deep theoreticians and responsible practical researchers formed the dynamite symmetrically doubled-ICA team.

Special thanks go out to some international experts in ICA: Mark Girolami, Michael Lewicki, Jean-François Cardoso, Russ Lambert and Bert-Uwe Köhler for collaborations, superb discussions and comments. In general, this book would have been impossible without the help of so many researchers working in this field. I appreciate their valuable discussions and comments during conferences and via email and fax.

I am indebted to many organizations for their financial support and general guidance: Max-Planck-Gesellschaft, AG fehlertolerantes Rechnen, Carnegie Mellon University, Alex Waibel's speech group, Daimler-Benz fellowship, Deutscher Akademischer Austauschdienst (DAAD) fellowship and Deutsche Forschungsgemeinschaft (DFG) postdoc fellowship.

Individual thanks go out to alumni of Club Thesis (Olivier, Michael, Marni, and K.T.) for pushing the work, and many other CNL-ers for making my stay at the Salk as pleasant as possible. Special thanks go out to Tim for constant support from Berlin and my godly woman Jee Hea for her caring love and wise thoughts. 


\section{List of Figures}

1.1 Entropy as a function of the probability $\mathrm{P}(\mathrm{x})$ for the coin tossing experiment.

1.2 Marginal entropies $H(X)$ and $H(Y)$, joint entropy $H(X, Y)$, conditional entropy $H(X \mid Y)$ and the mutual information $I(X ; Y)$. 10

$\begin{array}{lll}1.3 & \text { Simple single neuron. } & 15\end{array}$

$\begin{array}{lll}1.4 & \text { Oja's learning rule applied in a single neuron. } & 16\end{array}$

$\begin{array}{lll}1.5 & \text { Single layer feedforward neural network. } & 17\end{array}$

$\begin{array}{ll}1.6 & \text { A linear neural network with two neurons. } \\ \end{array}$

2.1 The instantaneous mixing and unmixing model. 30

2.2 Scatter plot of mixing and unmixing signals using PCA and ICA. 33

2.3 Original sources, mixed signals and recovered signals using PCA and ICA. 34

2.4 Top: logistic function and bottom: the derivate of the logistic function. 37

2.5 Left: p.d.f of a speech signal. Right: p.d.f. of a music signal. 38

2.6 Estimated densities for the extended infomax learning rule with $\mu_{i}=\{0 \cdots 2\}$.

2.7 Density model for the super-Gaussian distribution. The superGaussian model has a slightly heavier tail than the normal density.

$2.8 p(u)$ as a function of $b$. For $b=0$ the density estimate is suited to separate super-Gaussian sources. If for example $b=2$ the density estimate is bimodal and therefore suited to separated sub-Gaussian sources.

2.9 The kurtosis value is shown as a function of the shaping parameter $\mu$ and $b$ ( $\mu$ for the Pearson density model and $b$ for the hyperbolicCauchy density model). Both models approach $k_{4}=-2$ as the shaping parameter increases. The kurtosis for the Pearson model is strictly negative except for $\mu=0$. The kurtosis for the hyperbolicCauchy model ranges from positive to negative so that we may use this single parametric model to separate signals with sub- and super-Gaussian densities. 
2.10 Error measure $E$ in eq.2.69 for the separation of 10 sound sources. The upper curve is the performance for extended infomax and the lower curve shows the performance for the original infomax.

2.11 Performance matrix $\mathbf{P}$ for the separation of 20 sources using the original infomax algorithm after normalizing and reordering. Most super-Gaussian sources were recovered. However, the three subGaussian sources $(17,18,19)$, the Gaussian source $(20)$ and two superGaussian sources $(7,8)$ remain mixed and alias in other sources. In total, 14 sources were extracted and 6 channels remained mixed (see Table 2).

2.12 Performance matrix $\mathbf{P}$ for the separation of 20 sources using the extended infomax algorithm after normalizing and reordering. $\mathbf{P}$ is approximately the identity matrix which indicates nearly perfect separation.

2.13 For a fixed metric, the gradient in the orthonormal space exhibits optimal convergence because it points to the center of the solution.

2.14 P.d.f.s of source with a low and a high kurtosis.

2.15 Error measure given the number of iterations. Convergence is shown for three different nonlinearities.

4.1 The feedback architecture of eq.4.13, which is used to separate and deconvolve signals.

4.2 Learned Filters and time-delays for the full feedback system. 91

4.3 Entropy as a function of the delays $D_{12}$ and $D_{21}$. 92

4.4 The feedforward architecture used to separate and deconvolve signals. 93

4.5 The pole-zero diagram of a non-minimum phase mixing system. $\quad 95$

4.6 Learned Filters $W(z)$ for the feedforward system. 96

$\begin{array}{lll}4.7 & \text { Cartoon of the cocktail party problem. } & 97\end{array}$

4.8 Microphone outputs of one voice with background music recorded in a normal office room.

4.9 FIR 128-tap filters that unmixed and deconvolved the speech and music signals.

4.10 FIR 1024-tap filters that unmixed and deconvolved the speech and music signals.

4.11 Microphone outputs of two speakers recorded in a normal office room. 100

4.12 Room recordings from Yellin and Weinstein (1996).

4.13 An alternative multichannel deconvolution method. A complexvalued ICA algorithm is applied to each frequency bin of the observed data. The resulting unmixed blocks are rescaled and reordered and transformed back to the time domain. 
5.1 Illustration of basis vectors in a two-dimensional data space with two 2 sparse sources (top) or three sparse sources (bottom), (a) PCA finds orthogonal basis vectors and (b) ICA representation finds independent basis vectors. (c) ICA cannot model the data distribution adequately with three sources but (d) the overcomplete ICA representation finds 3 basis vectors that match the underlying data distribution (see Lewicki and Sejnowski).

5.2 Demonstration of the separation of three speech signals from two mixtures. (Top row) The time course of 3 speech signals. (Middle row) Two observations of three mixed speech signals. (Bottom row) The inferred speech signals.

5.3 Left: Two-dimensional scatter plot of the two mixed signals. The three basis vectors were randomly initialized. Right: After convergence the learned basis functions are shown as arrows along the three speech signals. The learned basis vectors may be permuted and have a different sign.

5.4 Signal to noise ratio (SNR) as a function of noise-level $l .{ }^{*}=$ speech signal $1 ;+=$ speech signal 2 and $o=$ speech signal 3.

5.5 Blind separation of speech and music. (Top row) The time course of 2 speech signals and a music signal. (Middle row) These were mixed into two observations. (Bottom row) The inferred speech and music signals.

6.1 Mixing and unmixing model for nonlinear ICA. 125

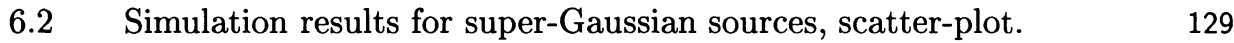

6.3 Simulation results using flexible nonlinearities.

6.4 Simulation results using flexible nonlinearities, time-course representation.

6.5 Example for the linearization approach to nonlinear ICA.

6.6 All possible combinations between the five outputs $u_{i}$.

7.1 The location of the electrodes for 14 channels according to the International 10-20 System.

7.2 A 10-sec portion of the EEG time series with prominent alpha rhythms $(8-21 \mathrm{~Hz})$.

7.3 The 14 ICA components extracted from the EEG data in figure 7.2. 151

7.4 Ratio of power near $60 \mathrm{~Hz}$ over 14 channels for EEG data, original infomax and extended infomax.

7.5 EEG data set with 23 channels including 2 EOG channels. At around 4-5 sec and 6-7 sec artifacts from severe eye blinks contaminate the data set.

7.6 Extended infomax ICA components derived from the EEG recordings in figure 7.5. The eye blinks are clearly concentrated in component 1 . Component 14 contains the steady state signal.

7.7 Artifact removal using the extended infomax algorithm.

7.8 Time course of the five artifactual components only.

7.9 Scalp map of 4 artifactual components. 
7.10 Time course of 10 randomly selected voxels recorded near the visual cortex.

7.11 The same voxels after ICA transformation. 160

$\begin{array}{lll}7.12 & \text { Brain maps of activations using different separation techniques. } & 161\end{array}$

7.13 Comparison of different methods with respect to the Stroop task reference function.

8.1 Linear image synthesis model with causes, basis functions and observed image patches.

8.2 Cartoon of a linear image synthesis model. $\quad 169$

8.3 Cartoon of an image recognition model. 170

8.4 ICA on natural images using extended infomax. 171

9.1 A simple example for classifying an ICA mixture model. There are 2 ICA classes (+) and (o), each class was generated by two independent variables, 2 bias terms and 2 basis vectors. Class (o) was generated by 2 uniform distributed sources as indicated next to the data class. Class (+) was generated by 2 Laplacian distributed sources with a sharp peak at the bias and heavy tails.

9.2 An example of classification of a mixture of independent components. There are 4 different classes, each generated by two independent variables and bias terms. The algorithm is able to find the independent directions (basis vectors) and bias terms for each class. 182 
List of Tables

2.1 Simulation results with 10 sources $\quad 51$

2.2 Simulation results with 20 sources 53

2.3 Robustness testing simulation with 10 sources 60

4.1 Speech recognition results [recognition rate in \%]. 101 


\section{Abbreviations and Symbols}

\section{Abbreviations}

ARMA

BCM

BSS

CDMA

c.d.f.

c.g.f.

DCA

EEG

EOG

ERP

FIR

fMRI

ICA

IIR

iff

infomax

$\mathrm{KL}$

MAP

MEG

m.g.f.

MLE

PCA

PET

p.d.f.

QPSK

RV

SOM

SOFM

SVD autoregressive moving average

Bienenstock, Copper and Moore theory of synaptic plasticity blind source separation code division multiple access cumulative density function cumulant generating function dynamic component analysis electroencephalographic electrooculographic event-related potentials

finite impuls response functional magnetic resonance imaging independent component analysis infinite impuls response if and only if principle of information maximization preservation Kullback-Leibler maximum a posteriori magnetoencephalography moment generation function maximum likelihood estimation principal component analyis positron emission tomography probability density function quadrature phase shift keying random variable self-organizing maps self-organizing feature maps singular value decomposition 
TDD

time-delayed decorrelation

\section{Symbols}

Vectors, matrices and high-dimensional matrices are-written in boldface. Usually vectors are lower-case type and matrices are higher-case type.
A
$\mathbf{A}(z)$
mixing matrix with elements $a_{i j}$
$A_{x}$
mixing matrix of filters with elements $A_{i j}(z)$
a
set of possible outcomes $\left\{a_{1}, a_{2}, \cdots\right\}$
b bias term
C covariance matrix
$C($.$) \quad cost function$
$c_{i}$
i-th order cumulant
$\mathbf{D}$
diagonal matrix
$D(. \|$.
Kullback-Leibler divergence
$D_{i j}$
mixing time-delay from channel $i$ to channel $j$
$d_{i j}$ unmixing time-delay from channel $i$ to channel $j$
$d_{i}, r_{i}$ inverse of scale $d_{i}$ and slope $r_{i}$ parameters for inverting the nonlinear transfer function
$\mathbf{E}$ matrix of which columns are eigenvectors of the covariance matrix expected value mean-squared error nonlinear activation function an arbitrary function
$H($. coefficients of the polynomial expansion of the transfer function nonlinearity
$h_{i}$
$h($.
$h_{i k}$
$\mathbf{i}_{\mathbf{s}}$
I
$I($.
$J($.
$\mathbf{J}$
K
$k_{i}$
$\mathbf{K}$
$L($.
$M$
$m_{i}$
$N$
$N_{X}$
differential entropy
orthogonal hermite polynomials
nonlinear inverse transfer function
coefficients of the polynomial expansion of the flexible nonlinearity information symbols
identity matrix
mutual information
negentropy
Jacobian matrix
number of classes
switching moments for sub- and super-Gaussian densities
diagonal matrix with elements $k_{i}$
log-likelihood function
number of sources
i-th order central moment
number of sensors
number of elements in $X$ 


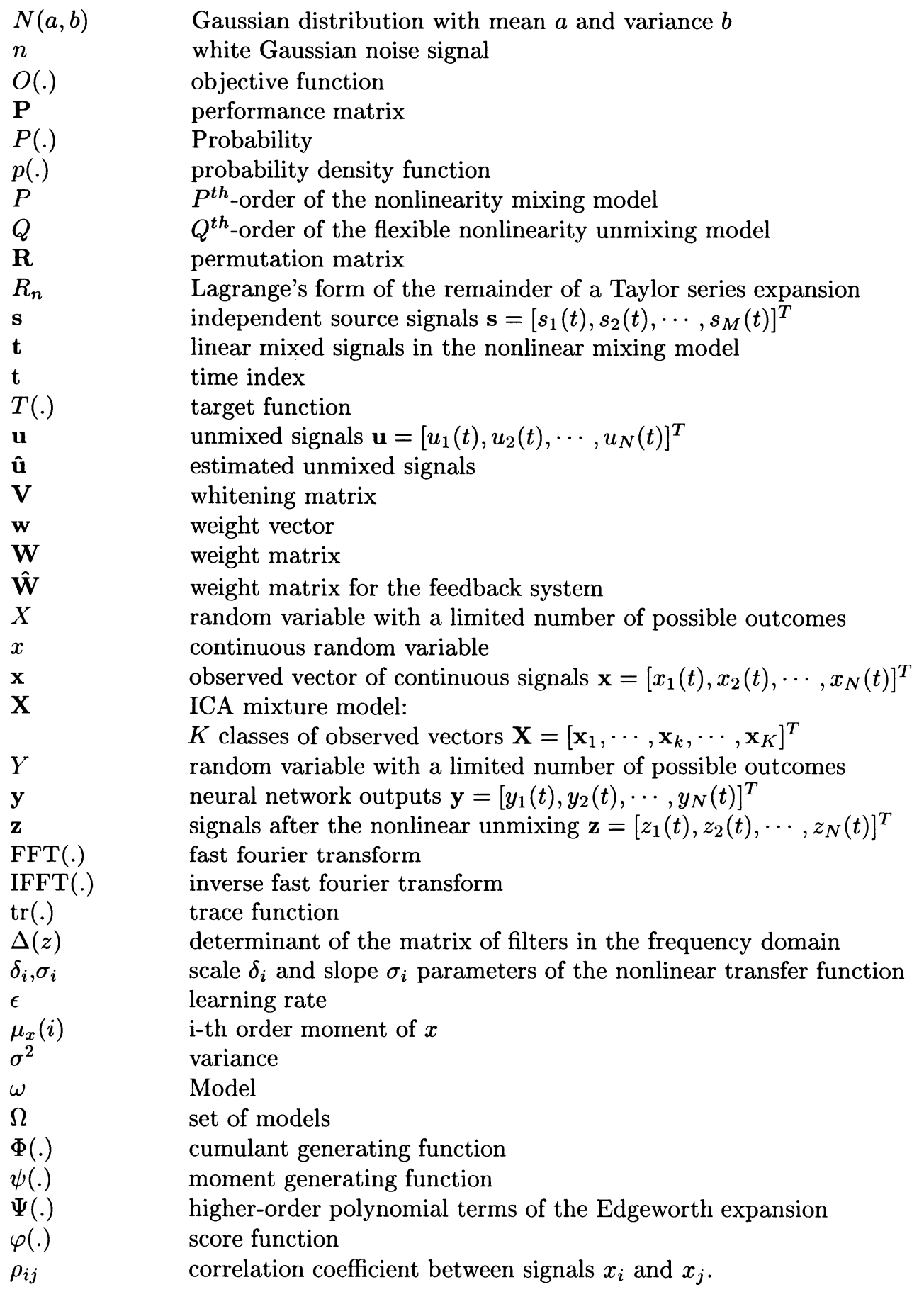


INTRODUCTION

\author{
"Begin at the beginning," the King said, gravely, " and go on \\ till you come to the end; then stop." \\ Lewis Carroll
}

\title{
A new star is born: ICA
}

Recently, blind source separation by Independent Component Analysis (ICA) has received attention because of its potential applications in signal processing such as in speech recognition systems, telecommunications and medical signal processing.

The goal of blind source separation (BSS) is to recover independent sources given only sensor observations that are linear mixtures of independent source signals. The term blind indicates that both the source signals and the way the signals were mixed are unknown. Independent Component Analysis (ICA) is a method for solving the blind source separation problem. It finds a linear coordinate system (the unmixing system) such that the resulting signals are statistically independent. In contrast to correlation-based transformations such as Principal Component Analysis (PCA), ICA not only decorrelates the signals (2nd-order statistics) but also reduces higherorder statistical dependencies. In other words, ICA is a method for finding a linear non-orthogonal co-ordinate system in any multivariate data. The directions of the axes of this co-ordinate system are determined by both the second and higher order statistics of the original data. The goal is to perform a linear transform which makes the resulting variables as statistically independent from each other as possible.

Two different research communities have considered the analysis of independent components. On one hand, the study of separating mixed sources observed in an array of sensors has been a classical and difficult signal processing problem. The seminal work on blind source separation was by Herault and Jutten (1986) where they introduced an adaptive algorithm in a simple feedback architecture that was able to separate several unknown independent sources. Their approach has been further developed by Jutten and Herault (1991), Karhunen and Joutsensalo (1994), and Cichocki et al. (1994). Comon (1994) elaborated the concept of independent component analysis and proposed cost functions related to the approximate minimization of mutual information between the sensors.

In parallel to blind source separation studies, unsupervised learning rules based on information theory were proposed by Linsker (1992). The goal was to maximize the mutual information between the inputs and outputs of a neural network. This approach is related to the principle of redundancy reduction suggested by Barlow (1961) as a coding strategy in neurons. Each neuron should encode features that are 
as statistically independent as possible from other neurons over a natural ensemble of inputs; decorrelation as a strategy for visual processing was explored by Atick (1992). Nadal and Parga (1994) showed that in the low-noise case, the maximum of the mutual information between the input and output of a neural network implied that the output distribution was factorial; that is, the multivariate probability density function (p.d.f.) can be factorized as a product of marginal p.d.f.s. Roth and Baram (1996) and Bell and Sejnowski (1995) independently derived stochastic gradient learning rules for this maximization and applied them, respectively, to forecasting, time series analysis, and the blind separation of sources. Bell and Sejnowski (1995) put the blind source separation problem into an information-theoretic framework and demonstrated the separation and deconvolution of mixed sources. Their adaptive methods are more plausible from a neural processing perspective than the cumulant-based cost functions proposed Comon (1994). A similar adaptive method for source separation was proposed by Cardoso and Laheld (1996).

Other algorithms for performing ICA have been proposed from different viewpoints. Maximum Likelihood Estimation (MLE) approaches to ICA were first proposed by Gaeta and Lacoume (1990) and elaborated by Pham et al. (1992). Pearlmutter and Parra (1996), MacKay (1996) and Cardoso (1997) showed that the infomax approach of Bell and Sejnowski (1995) and the maximum likelihood estimation approach are equivalent. Girolami and Fyfe (1997c) motivated by informationtheoretic indices for Exploratory Projection Pursuit (EPP) used marginal negentropy as a projection index and showed that kurtosis-seeking projection pursuit will extract one of the underlying sources from a linear mixture. A multiple output EPP network was developed to allow full separation of all the underlying sources (Girolami and Fyfe, 1997b). Nonlinear PCA algorithms for ICA which have been developed by Karhunen and Joutsensalo (1994), Xu (1993) and Oja (1997) can also be viewed from the infomax principle since they approximately minimize the sum of squares of the fourth-order marginal cumulants (Comon, 1994) and therefore approximately minimize the mutual information of the network outputs (Girolami and Fyfe, 1997d). Bell and Sejnowski (1995) have pointed out a similarity between their infomax algorithm and the Bussgang algorithm in signal processing and Lambert (1996) elucidated the connection between three different Bussgang cost functions. Lee et al. (1998a) show how the Bussgang property relates to the infomax principle and how all of these seemingly different approaches can be put into a unifying framework for the source separation problem based on an information theoretic approach.

The original infomax learning rule for blind separation by Bell and Sejnowski (1995) was suitable for super-Gaussian sources. An extension of the infomax algorithm of Bell and Sejnowski (1995) is presented in Lee et al. (1998b) that is able to blindly separate mixed signals with sub- and super-Gaussian source distributions. This was achieved by using a simple type of learning rule first derived by Girolami (1997b) by choosing negentropy as a projection pursuit index. Parameterized probability distributions that have sub- and super-Gaussian regimes were used to derive a general learning rule that preserves the simple architecture proposed by Bell and Sejnowski (1995), is optimized using the natural gradient by Amari (1998), and 
uses the stability analysis of Cardoso and Laheld (1996) to switch between sub- and super-Gaussian regimes.

Extensive simulations have been performed to demonstrate the power of the learning algorithm. However, instantaneous mixing and unmixing simulations are toy problems and the challenge lies in dealing with real world data. Makeig et al. (1996) have applied the original infomax algorithm to EEG and ERP data showing that the algorithm can extract EEG activations and isolate artifacts. Jung et al. (1998a) show that the extended infomax algorithm is able to linearly decompose EEG artifacts such as line noise, eye blinks, and cardiac noise into independent components with suband super-Gaussian distributions. McKeown et al. (1998b) have used the extended ICA algorithm to investigate task-related human brain activity in fMRI data. By determining the brain regions that contained significant amounts of specific temporally independent components, they were able to specify the spatial distribution of transiently task-related brain activations. Other potential applications may result from exploring independent features in natural images. Bell and Sejnowski (1997) suggest that independent components of natural scenes are edge filters. The filters are localized, mostly oriented and similar to Gabor like filters. The outputs of the ICA filters are sparsely distributed. Bartlett and Sejnowski (1997) and Gray et al. (1998) demonstrate the successful use of the ICA filters as features in face recognition tasks and lipreading tasks respectively. In a similar manner, Bell and Sejnowski (1996) applied the infomax algorithm to learning higher-order structure of a natural sound.

For these applications, the instantaneous mixing model may be appropriate because the propagation delays are negligible. However, in real environments substantial time-delays may occur and an architecture and algorithm is needed to account for the mixing of time-delayed sources and convolved sources. The multichannel blind source separation problem has been addressed by Yellin and Weinstein (1994) and Nguyen-Thi and Jutten (1995) and others based on $4^{\text {th }}$-order cumulants criteria. An extension to time-delays and convolved sources from the infomax viewpoint using a feedback architecture was developed by Torkkola (1996a). Lee et al. (1997a) extended the blind source separation problem to a full feedback system and a full feedforward system. The feedforward architecture allows the inversion of nonminimum phase systems. In addition, the rules are extended using polynomial filter matrix algebra in the frequency domain (Lambert, 1996). The proposed method can successfully separate voices and music recorded in a real environment. Lee et al. (1997b) showed that the recognition rate of an automatic speech recognition system was increased after separating the speech signals.

Since ICA is restricted and relies on several assumptions researchers have started to tackle a few limitations of ICA. One obvious but non-trivial extension is the nonlinear mixing model. In (Hermann and Yang, 1996; Lin and Cowan, 1997; Pajunen, 1996) nonlinear components are extracted using self-organizing-feature-maps (SOFM). Other researchers (Burel, 1992; Lee et al., 1997c; Taleb and Jutten, 1997; Yang et al., 1997; Hochreiter and Schmidhuber, 1998) have used a more direct extension to the previously presented ICA models. They include certain flexible nonlinearities in the mixing model and the goal is to invert the linear mixing matrix 
as well as the nonlinear mixing. More recently, Hochreiter and Schmidhuber (1998) have proposed low complexity coding and decoding approaches for nonlinear ICA. Another limitation is the under-determined problem in ICA, i.e. having less sensors than sources. Lee et al. (1998c) demonstrated that an overcomplete representation (Lewicki and Sejnowski, 1998b) of the data can be used to learn non-square mixing matrices and to infer more sources than sensors. The overcomplete framework also allows additive noise in the ICA model and can therefore be used to separate noisy mixtures.

There is now a substantial amount of literature on ICA and BSS. Reviews of the different theories can be found in Cardoso and Comon (1996); Cardoso (1998b); Lee et al. (1998a) and Nadal and Parga (1997). Several neural network learning rules are reviewed and discussed by Karhunen (1996); Cichocki and Unbehauen (1996) and Karhunen et al. (1997a).

ICA is a fairly new and a generally applicable method to several challenges in signal processing. It reveals a diversity of theoretical questions and opens a variety of potential applications. Successful results in EEG, fMRI, speech recognition and face recognition systems indicate the power and optimistic expectations in the new paradigm.

\section{Organization of the book}

This book is partitioned into Theory and Applications of ICA. The theory part of ICA includes(basic theory (chapter 1), ICA (chapter 2), unifying approach for ICA (chapter 3), multichannel deconvolution (chapter 4), overcomplete ICA (chapter 5) and nonlinear ICA (chapter 6).

- Chapter 1 starts with an introduction to Bayesian probability theory, information theory, artificial neural networks and higher-order statistics. Only some basics and some properties that are needed are recalled to further derive and explain the methods and algorithms performing ICA.

- Chapter 2 states the ICA problem and explains why decorrelation-based methods fail to separate sources and why higher-order methods are needed to solve this problem. The unsupervised learning algorithm by Bell and Sejnowski (1995) is analyzed that is able to blindly separate mixed sources with super-Gaussian distributions. An extension of this algorithm is presented that is able to blindly separate mixed signals with sub- and super-Gaussian source distributions (Girolami, 1997b; Lee et al., 1998b).

- Chapter 3 presents different theories recently proposed for ICA and show how they lead to the same iterative learning algorithm for blind separation of mixed independent sources. Those seemingly different theories are reviewed and put in an information theoretic framework to unify several lines of research.

- Chapter 4 deals with time-delayed and convolved sources, the multichannel blind deconvolution problem. Learning algorithms for the feedforward and feedback 
architecture are presented. These methods is applied to multisensory recordings and is able to separate voices and music recorded in real environments.

- Chapter 5 presents ICA results using an overcomplete representation (Lewicki and Sejnowski, 1998b). This generalization includes an additive noise model and allows the separation of more sources than mixtures.

- Chapter 6 tries to eliminate several constraints in the standard ICA formulation. The linear model is generalized by simple nonlinear mixing models for nonlinear ICA. A set of learning algorithms are derived and verified in simulations.

The applications part of ICA includes ICA for biomedical signal processing (chapter 7), feature extraction (chapter 8) and ICA for unsupervised classification (chapter 9$)$.

- Chapter 7 presents examples on how ICA can be used to (1) isolate artifacts in EEG recordings and (2) to detect transiently activated brain signals in fMRI experiments.

- Chapter 8 demonstrates how ICA can extract features from natural images. Those features can be used in recognition systems such as face recognition and lipreading systems to improve overall performance.

- Chapter 9 shows how the ICA algorithm, can be further embedded in an expectation maximization framework with the goal to classify mixtures of ICA models. This approach is an extension of the Gaussian mixture model for non-Gaussian priors. Results on several classification benchmarks demonstrate that ICA cluster models can improve many classification results.

Chapter 10 gives conclusions by summarizing the main results and discussing future challenges in ICA research. 